\title{
Analyses on Thiophene-Based Donor-Acceptor Semiconducting Polymers toward Designing Optical and Conductive Properties: A Theoretical Perspective
}

Toru Matsui, ${ }^{a}$ Yutaka Imamura, ${ }^{a}$ Itaru Osaka, ${ }^{b}$ Kazuo Takimiya ${ }^{b}$, and Takahito Nakajima ${ }^{a}$ *

${ }^{a}$ Computational Molecular Science Research Team, RIKEN Advanced Institute for Computational Science, 7-1-26, Minatojima-minami-machi, Chuo-ku, Kobe, Hyogo 650-0047, Japan

${ }^{b}$ Emergent Molecular Function Research Group, RIKEN Center for Emergent Matter Science (CEMS), Wako, Saitama 351-0198, Japan

* Corresponding author. E-mail: nakajima@,riken.jp. Tel: +81-78-940-5534 
Supporting data 1: Detailed data for Tables 1, 2 and Figure 4.

Table S1: Computed energy levels at frontier orbitals of $N$-mer.

\begin{tabular}{lcccccccc}
\hline \multicolumn{1}{c}{ HOMO } & \multicolumn{5}{c}{ LUMO } \\
\multicolumn{1}{c}{} & 1 & 2 & 3 & 4 & 1 & 2 & 3 & 4 \\
\hline DPP & -5.396 & -5.251 & -5.204 & -5.197 & -2.955 & -3.116 & -3.177 & -3.217 \\
BTz & -5.434 & -5.158 & -5.090 & -5.061 & -2.893 & -2.989 & -3.030 & -3.048 \\
TzTz & -5.429 & -5.219 & -5.162 & -5.139 & -2.403 & -2.672 & -2.734 & -2.760 \\
NTz & -5.517 & -5.245 & -5.190 & -5.165 & -3.112 & -3.173 & -3.213 & -3.211 \\
TPD & -5.570 & -5.309 & -5.250 & -5.226 & -2.524 & -2.781 & -2.838 & -2.859 \\
NDI & -6.600 & -6.033 & -5.912 & -5.829 & -3.576 & -3.646 & -3.675 & -3.700 \\
\hline
\end{tabular}

Note: Unit is given in $\mathrm{eV}$.

Table S2: Computed energy levels at frontier orbitals of $N$-mer appeared in Figure 3.

\begin{tabular}{lcccccccc}
\hline \multicolumn{1}{c}{ HOMO } & \multicolumn{5}{c}{ LUMO } \\
\multicolumn{1}{c}{$N$} & 1 & 2 & 3 & 4 & 1 & 2 & 3 & 4 \\
\hline DPP & -5.396 & -5.251 & -5.204 & -5.197 & -2.955 & -3.116 & -3.177 & -3.217 \\
isoDPP & -5.682 & -5.358 & -5.319 & -5.303 & -2.993 & -3.099 & -3.142 & -3.158 \\
$\operatorname{TTD}(\mathrm{O})$ & -5.740 & -5.416 & -5.354 & -5.328 & -3.344 & -3.432 & -3.473 & -3.492 \\
$\mathrm{TTD}(\mathrm{S})$ & -5.722 & -5.406 & -5.343 & -5.313 & -3.541 & -3.624 & -3.666 & -3.679 \\
$\mathrm{TTD}(\mathrm{Se})$ & -5.713 & -5.398 & -5.337 & -5.305 & -3.550 & -3.634 & -3.676 & -3.688 \\
\hline
\end{tabular}

Note: Unit is given in $\mathrm{eV}$. 
Table S3: Detailed data for Figure 5.

\begin{tabular}{ccccc}
\hline & $\rho_{\text {HOMO }}$ & $\beta_{\text {HOMO }}$ & $\rho_{\text {LUMO }}$ & $\beta_{\text {LUMO }}$ \\
\hline BTz & 0.0373 & -0.219 & 0.0121 & -0.071 \\
TzTz & 0.0306 & -0.180 & 0.0340 & -0.200 \\
NTz & 0.0355 & -0.208 & 0.0082 & -0.048 \\
TPD & 0.0358 & -0.210 & 0.0266 & -0.156 \\
NDI & 0.0944 & -0.554 & 0.0122 & -0.071 \\
DPP & 0.0214 & -0.126 & 0.0218 & -0.128 \\
isoDPP & 0.0387 & -0.227 & 0.0139 & -0.082 \\
FFD & 0.0445 & -0.261 & 0.0131 & -0.077 \\
TTD & 0.0296 & -0.174 & 0.0128 & -0.075 \\
SSD & 0.0435 & -0.255 & 0.0126 & -0.074 \\
\hline
\end{tabular}

Note: $\beta_{\text {HOMO }}$ or $\beta_{\text {LUMO }}$ is obtained by the fitting 
Supporting data 2: Molecular orbital difference between monomer and dimer for each compound.

BTz-4T

$N=1$, HOMO

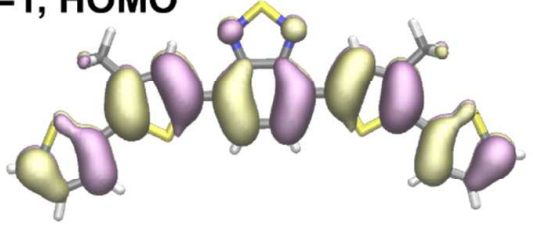

$\mathbf{N}=2$, HOMO

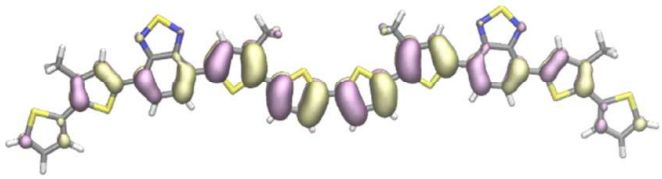

TzTz-4T

$N=1$, HOMO

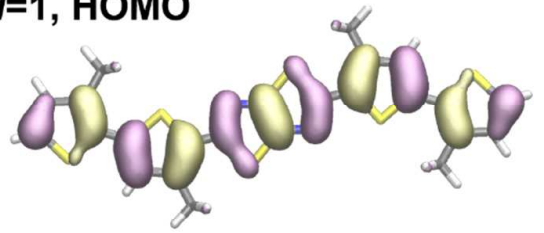

$\mathrm{N}=2$, HOMO

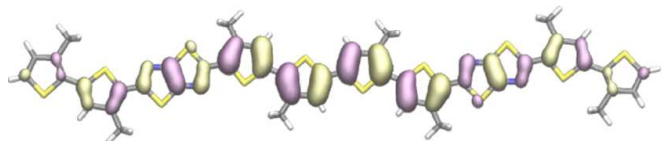

$N=1$, LUMO

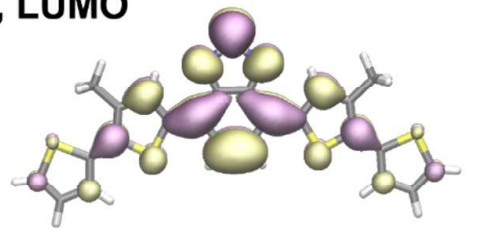

$N=2$, LUMO
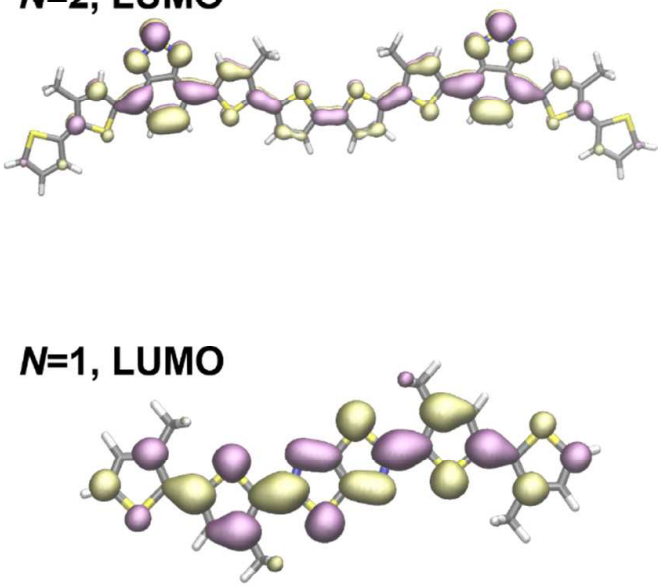

N=2, LUMO

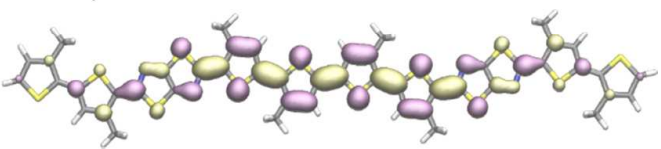


NTz-4T

$$
N=1 \text {, HOMO }
$$

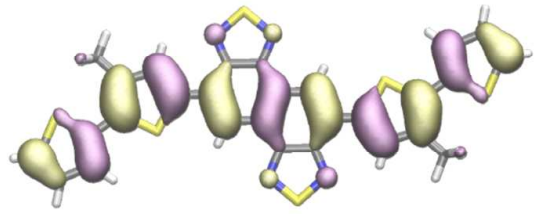

$\mathbf{N}=2$, НОМО

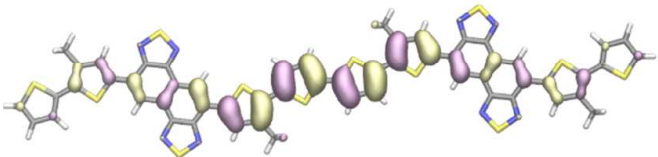

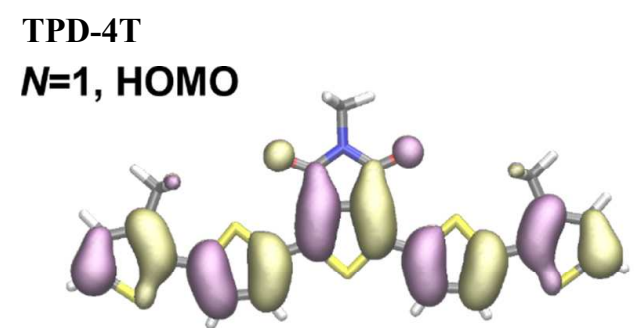

$\mathbf{N}=2$, HOMO

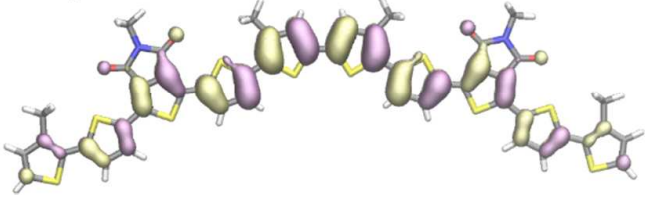

NDI-2T

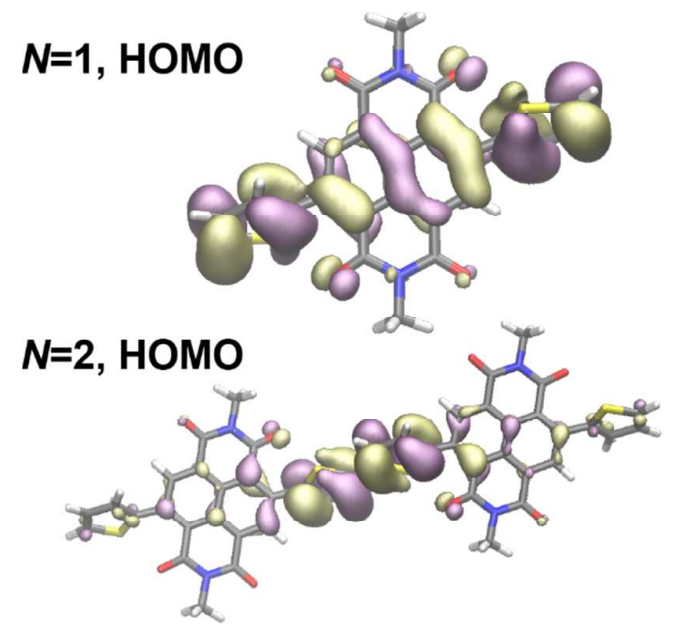

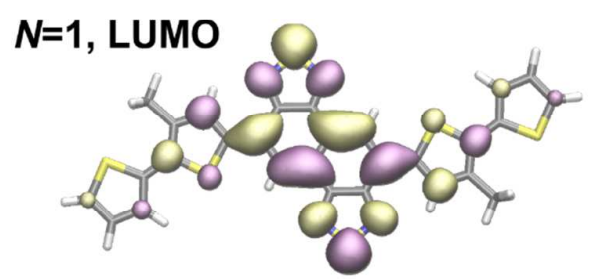

$\mathbf{N}=2$, LUMO

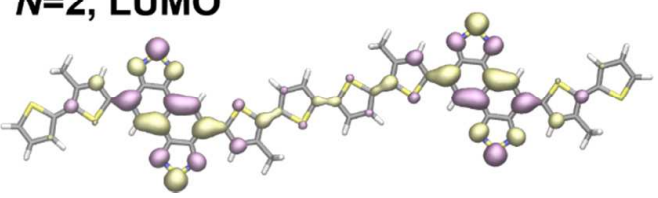

$N=1$, LUMO

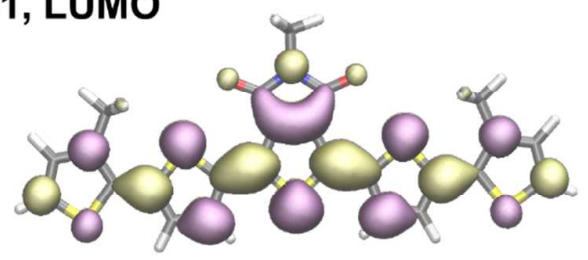

$\mathbf{N}=2$, LUMO
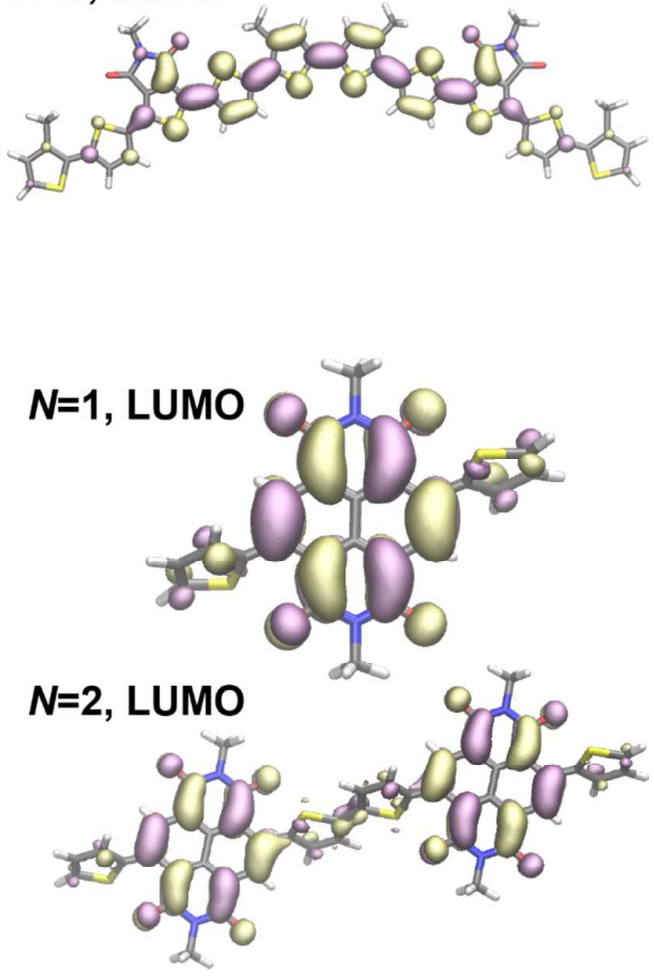
DPP-4T

N=1, HOMO

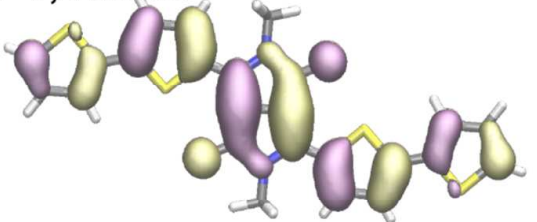

$\mathbf{N}=2$, HOMO

$N=1$, LUMO

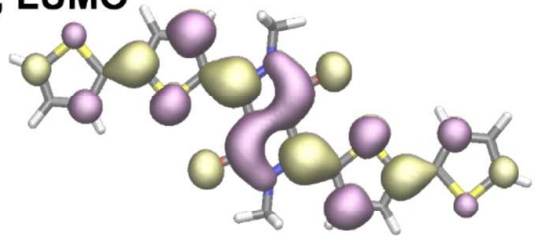

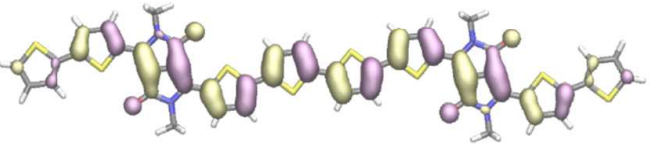

$\mathbf{N}=\mathbf{2}$, LUMO

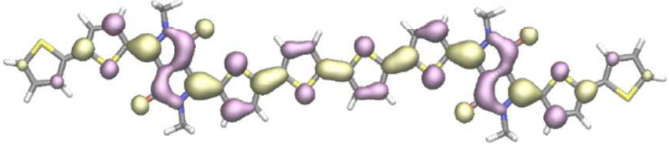

isoDPP-4T

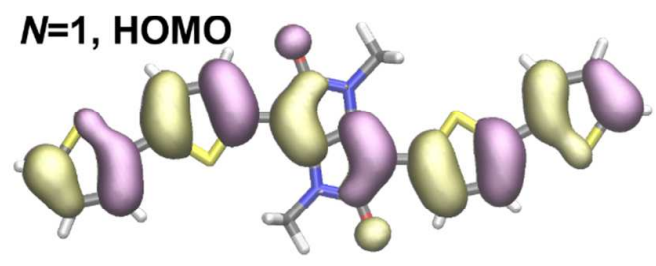

$\mathbf{N}=\mathbf{2}$, HOMO

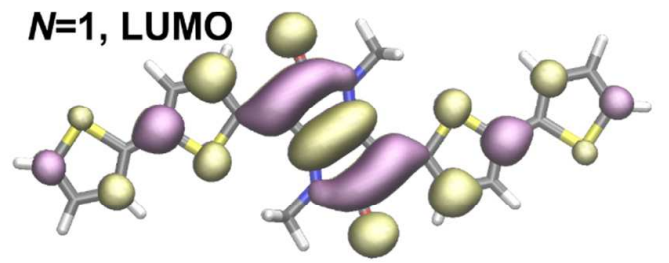

$\mathbf{N}=\mathbf{2}$, LUMO

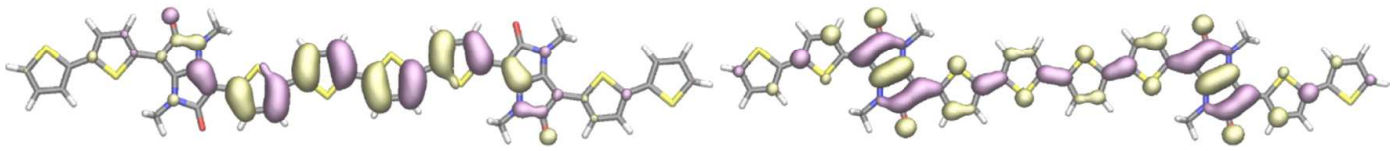

FFD-4T

$N=1$, HOMO

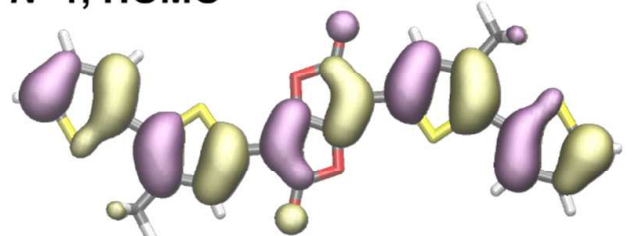

$N=2$, HOMO

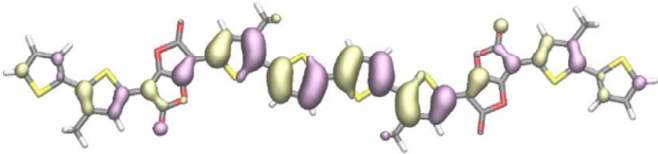

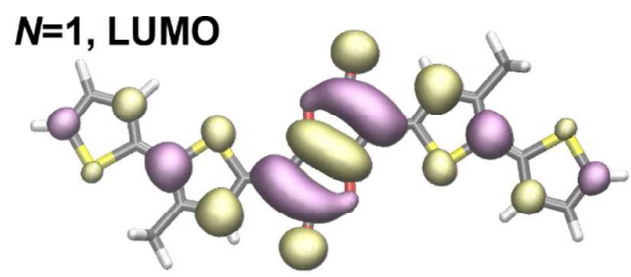

$N=2$, LUMO

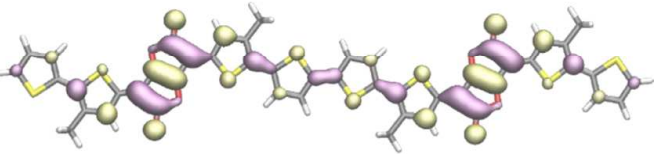


TTD-4T
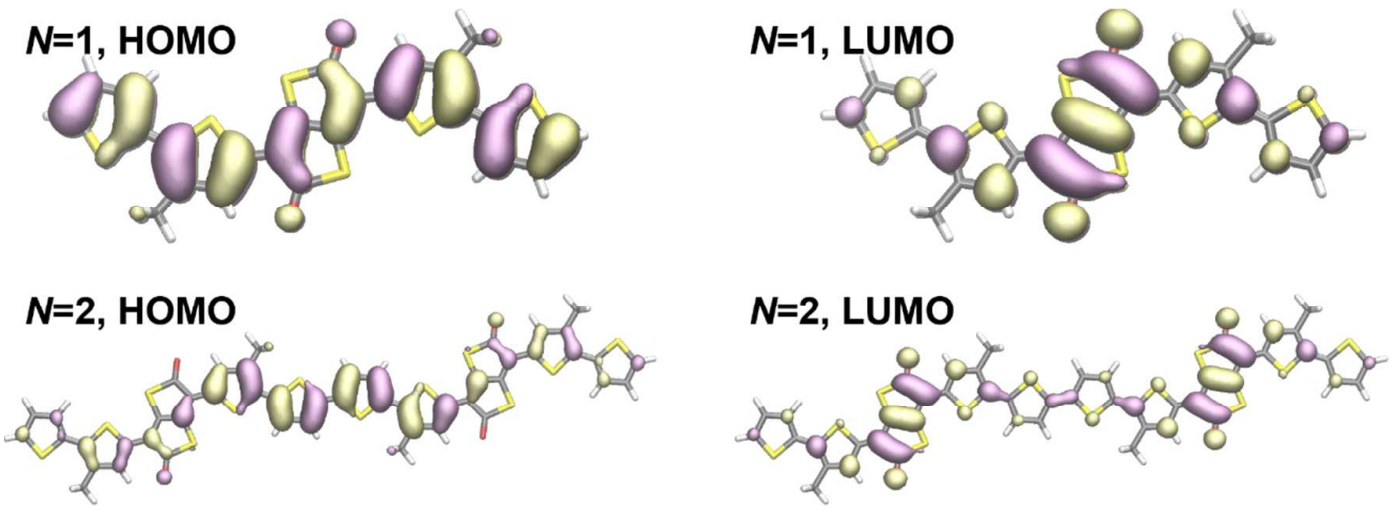

SSD-4T

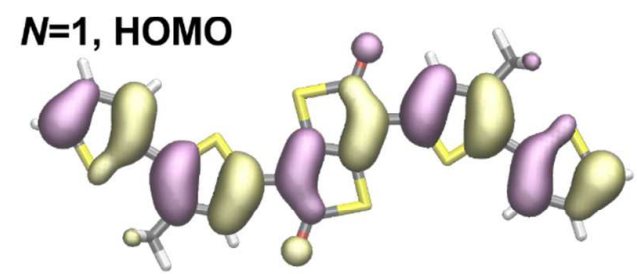

$\mathbf{N}=2$, НОМО

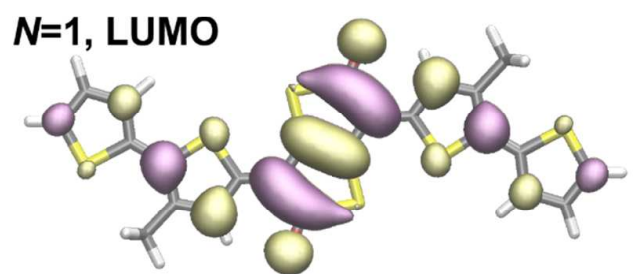

$N=2$, LUMO
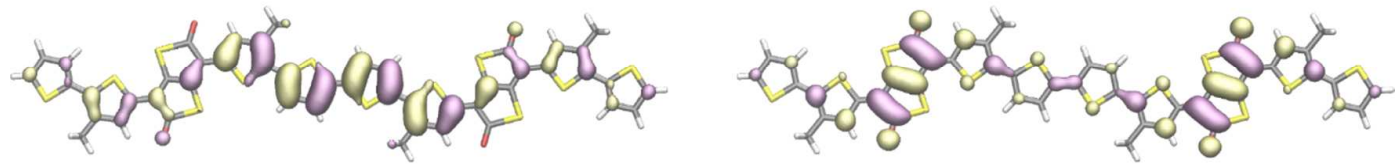